\title{
Características De Salud Y Seguridad En Trabajadores De Una Empresa Automotriz
}

\author{
Mayra Rita Rodríguez-Morales, Pasante en Servicio Social
}

Licenciatura en Enfermería, Universidad Autónoma de Querétaro, México

Nancy Viridiana Ruiz-Vargas, ME

Kellogg de México, Querétaro, México

Ruth Magdalena Gallegos-Torres, Dra. en C.S.

Universidad Autónoma de Querétaro, México

Doi:10.19044/esj.2020.v16n24p336 URL:http://dx.doi.org/10.19044/esj.2020.v16n24p336

\section{Resumen}

Introducción: El lugar de trabajo es más que el espacio donde los trabajadores prestan sus servicios, por lo tanto, las condiciones de estos lugares pueden afectar de manera significativa el desempeño y la productividad de los empleados. Objetivo: Describir las características de salud y seguridad presentes en los trabajadores de una empresa automotriz de la ciudad de Querétaro, a través de un mapeo de riesgos utilizando el método FINE. Metodología. Estudio transversal, descriptivo, realizado en una empresa de giro automotriz implementando un mapa de riesgo, siguiendo el método FINE. Se consideraron trabajadores de sexo indistinto, con antigüedad mínima de 6 meses, de las áreas de mantenimiento y reparación, que aceptaran participar en el estudio y firmaran el consentimiento informado. El proyecto fue evaluado y registrado ante un Comité de Investigación y Bioética. El análisis de los datos se realizó mediante estadística descriptiva. Se cuidaron los principios éticos de la investigación en humanos. Resultados: La zona evaluada consta de un total de 42 individuos, siendo de ellos en su mayoría hombres, con un 71\% (30), rango de 18 a 59 años de edad. La estática postural despunta con un 71\% (30) de los trabajadores. Se encontró la presencia de un riesgo moderado y grave. Conclusión:Resulta relevante abordar a los trabajadores desde un aspecto personal y familiar, puesto que estos factores resultan de importancia debido a que pueden potencializar la fuente generadora de un daño a la salud por causa de los diversos riesgos existentes en los lugares de trabajo.

Palabras clave: Método FINE, Prevención, Riesgos, Salud, Seguridad, Trabajadores 


\title{
Health And Safety Characteristics In Workers Of An Automotive Company
}

\author{
Mayra Rita Rodriguez-Morales, Pasante en Servicio Social \\ Licenciatura en Enfermería, Universidad Autónoma de Querétaro, México \\ Nancy Viridiana Ruiz-Vargas, $\mathrm{ME}$ \\ Kellogg de México, Querétaro, México
}

Ruth Magdalena Gallegos-Torres, Dra. en C.S.

Universidad Autónoma de Querétaro, México

\begin{abstract}
Introduction: The workplace is something else than just a space where workers provide their services. Therefore, conditions prevailing in these places may significantly affect employee performance and productivity. Objective: To describe health and safety features prevailing in the workers of an automotive company in Queretaro City by mapping risks, using the FINE method. Methodology. Transversal, descriptive survey applied at an automotive branch company implementing a risk map, following the FINE method. It will cover indistinct gender workers with a minimum of 6-month seniority from the maintenance and repair areas, who shall accept to take part in the study and sign an informed consent. The project was evaluated and registered with the Research and Bioethics Committee. Data analysis was made by descriptive statistics. The ethical principles of research on humans were taken into account. Results: The evaluate zone has a total of 42 individuals, mostly men, with a $71 \%$ (30), range of 18 to 59 years of age. Static posture deploys with $71 \%$ (30) of the workers. The presence of moderate and severe risks has been found. Conclusion: Addressing workers from a personal and family perspective is relevant; these factors are important as they may strengthen a health damage-producing source caused by several risks existing at work places
\end{abstract}

Keywords: FINE Method, Prevention, Risks, Health, Safety, Workers

\section{Introducción:}

El lugar de trabajo es más que el espacio donde los trabajadores prestan sus servicios, por lo tanto, las condiciones que presentan pueden afectar de manera significativa el desempeño y la productividad de los empleados.

La Organización Internacional del Trabajo (OIT, 2015), expone que los sitios de trabajo implican riesgos para la salud de los individuos, mismos 
que son causados por las características del medio ambiente laboral, ya que sugieren el manejo de agentes biológicos, físicos, químicos y de seguridad, además de las condiciones generales de infraestructura, procedimientos de trabajo y producción, duración de las jornadas de trabajo, horarios de trabajo y forma de remuneración, organización y contenido de trabajo, sumado a los servicios sociales y de bienestar. Dependiendo de la exposición y condiciones de la persona, todos los anteriores pueden provocar enfermedades ocupacionales y agravar otros problemas de salud pública (OMS, 2017).

En este sentido, las empresas se ven en la obligación de reportar los accidentes de trabajo que padecen los empleados. Las enfermedades ocupacionales y los riesgos a la salud no están exentos en las compañías, industrias, etc., aumentando con esto el número de muertes, heridas y enfermedades relacionadas con el trabajo, lo que hace necesario desarrollar una cultura de seguridad preventiva.

Lo anterior, puede determinarse a través del uso de múltiples herramientas, siendo una de ellas el mapeo de riesgos, el cual permite establecer las características de salud y del ambiente de los trabajadores, apoyando con esto en la identificación de posibles factores de riesgo existentes para el desarrollo de lesiones o complicaciones relacionadas con el trabajo. A partir de contar con un referente informativo del fenómeno, se pueden crear de forma estratégica una serie de intervenciones de carácter preventivo entre las que se incluya la enseñanza a los trabajadores acerca de la práctica correcta a realizar dentro de las instalaciones de trabajo, con la finalidad de disminuir las estadísticas de riesgos laborales (Enciso Saldarriaga, López Sierra, \& Domínguez Ramírez, 2015).

Para que esto suceda, se vuelve prioritario involucrar: 1) a los responsables de los diferentes niveles de la organización para la implementación de diversos programas de salud y seguridad, basados en las normas oficiales mexicanas de la STPS (Secretaría del Trabajo y Previsión Social) y las que correspondan, 2) y al trabajador.

De lo anterior, el Diario Oficial de la Federación (Federación, 2018), en pro de la salud de los trabajadores, publicó la Norma Oficial Mexicana NOM-035-STPS-2018, que expidió la Secretaría del Trabajo y Previsión Social, denominada "Factores de riesgo psicosocial en el trabajoIdentificación análisis y prevención”, por medio de la que se establecen disposiciones que deberán adoptarse en los centros de trabajo, y así promover un entorno organizacional favorable, mediante acciones que impulsen el apoyo social, la difusión de la información y la capacitación, donde de igual manera, se deberá establecer por escrito, implantar, mantener y difundir la política de prevención de riesgos psicosociales, todo esto como parte de una serie de obligaciones del patrón de la empresa, así como también 
las obligaciones de los trabajadores para dar cumplimiento y colaborar en las medidas dispuestas por la norma.

La metodología de mapas de riesgo de trabajo seguro (SafeWork) de la OIT tiene el propósito de apoyar a las empresas en la valoración de sus condiciones de salud y seguridad en el trabajo, concretando así, las actividades de mejora continua y acciones que determinan estrategias para procurar ambientes de trabajo sanos y seguros (SIMAPRO-OIT, 2017).

El Sistema Integral de Medición y Avance de la Productividad de la OIT, refriere que la aplicación de estas metodologías promueve la gestión de la salud y la seguridad en el proceso de trabajo de todo tipo de organizaciones y sectores, no importando el nivel de la empresa, llámese micro, mediano o grande (SIMAPRO-OIT, 2017).

Con lo anterior, se coadyuva al fortalecimiento de la propia empresa y del trabajador, fomentando su autocuidado, obteniendo de esto, una disminución en cuanto a accidentes, promoción y aplicación del cumplimiento de marcos normativos por los cuales se rigen las empresas y de los procesos relacionados a la prevención de la seguridad y la salud de los empleados (SafeWork, 2016).

Los sectores en los que se ha aplicado la metodología son: la agroindustria de la caña de azúcar en Cuba y México, hotelería en turismo en Nuevo Vallarta, México; en el sector frutícola de Chile y de manera más acotada en algunas PYME de México.

La Organización Internacional del Trabajo (OIT), publicó el estudio “Prácticas de Trabajo Seguro en el Ingenio Pánuco”, en donde realizó la aplicación de la metodología de mapeo de riesgos en el sector azucarero, en el que se obtuvo como resultado una reducción del $36 \%$ de gravedad de accidentes del 2013 a 2015, esto, mediante la implementación de medidas estratégicas para el control de riesgos ante la identificación temprana de los mismos (OIT, 2015).

A su vez, en el estudio “Gestión de perfiles, mapas de riesgos y planes de acción para un Trabajo Seguro en la Industria Azucarera, 2017”, se señala que la industrialización del azúcar se caracteriza por su alta siniestralidad (Falcón \& Rojas, 2017). La industria presentaba en 2015 una tasa de 5,3 que es dos veces mayor que el promedio nacional, por lo tanto, la promoción del Trabajo Seguro fue uno de los ejes de acción de la asistencia técnica de la OIT a la agroindustria de la caña de azúcar en México y Cuba.

Por otra parte, la Secretaría de Salud Laboral y Medio Ambiente MCAUGT (Ferreras Remesal, López Ureña, Piedrabuena Cuesta, Oltra Pastor, \& Ruiz Folgado, 2011), en su manual de "Ergonomía y PYMES: Especial referencia al sector de talleres de reparación de automóviles”, señala que la prevención de riesgos laborales es uno de los indicadores más potentes para medir la calidad de las condiciones de trabajo y se relaciona ampliamente con 
la salud, el bienestar, la satisfacción y también la productividad. Así como la seguridad y la higiene han mejorado notablemente en los últimos años, los problemas asociados a unas condiciones ergonómicas inadecuadas del trabajo están adquiriendo una importancia creciente, esto es particularmente visible en los talleres de reparación de automóviles.

De acuerdo con Enrique Reyes Jiménez, jefe de Afiliación y Cobranza del Instituto Mexicano del Seguro Social (IMSS), de Aguascalientes, México, la industria automotriz se encuentra entre los lugares más peligrosos para trabajar, de acuerdo a las clasificaciones que realiza el IMSS, por los accidentes de trabajo y las enfermedades relacionadas con esta labor, es por eso que es uno de los giros que más eroga por la "Prima en el Seguro de Riesgos de Trabajo” (Álvarez Fernández, 2015).

El Instituto Nacional de Seguridad y Salud en el Trabajo en su Enciclopedia de Salud y Seguridad (INSHT, 2015), resume que la identificación, la medición y la descripción de los accidentes constituyen la base sobre la que se establece qué acciones emprender y quién debe encargarse de las mismas para reducir los riesgos. Si se demuestra que los accidentes frecuentes y graves están asociados a ciertos procesos, puede intentarse ajustar las características de los equipos, la maquinaria, las operaciones y los procedimientos de trabajo vinculados a dichos procesos.

A pesar de señalarse a la industria automotriz como un área relevante para abordar la salud de los trabajadores, en la literatura internacional y nacional, existen escasos estudios similares al que se propone, que arrojen información con respecto a las características, factores o riesgos de salud de los trabajadores de las empresas o bien que presenten resultados de la implementación de la metodología SafeWork, por lo que su contraste con otras investigaciones es complejo.

Así, se vuelve relevante el establecimiento de las características de salud y seguridad laborales de los trabajadores en una zona como la del estado de Querétaro, donde el desarrollo industrial es de alto impacto (Zepeda García \& García García, 2018), teniendo empresas de tipo alimentario, manufacturero, automotriz, entre muchas otras; con esto, se pueden observar posibles riesgos laborales.

Con proyectos realizados por el área de enfermería en el ámbito laboral, se puede reflejar la importancia del gremio en dos distintas áreas: la investigación en salud y del trabajo en el sector industrial, ya que los profesionales de esta índole pueden involucrarse tanto en aspectos de salud como de seguridad, actuando junto con un equipo multidisciplinario, orientando al personal e incentivando a crear una cultura de seguridad propia, favoreciendo la calidad de vida laboral.

La enfermera ocupacional juega un gran papel, ya que continuamente tiene que evaluar los aspectos de salud que giran en torno al trabajador, por lo 
que es relevante que no sólo se enfoque en la salud de cada individuo, sino en su ambiente y en la motivación del mismo para la adecuada protección y uso de medidas de seguridad (González Sánchez, 2011).

En México, la evidencia empírica ha evidenciado que la presencia de la enfermera industrial aún no es evidente en todas y cada una de las compañías nacionales o internacionales que existen, sin embargo, es una situación que va a la alta, dado que se reconoce la importancia del cuidado de la salud de los trabajadores, aplicando ante todo aspectos de prevención y promoción de la salud que favorezcan, además de la prevención de accidentes, la disminución de prácticas riesgosas y de igual manera el ausentismo; es así que, considerando las condiciones de trabajo en México, la intervención de los profesionales de enfermería se vuelve fundamental, ya que favorece a la industria al mantener en óptimas condiciones al personal operativo y dando cumplimiento a los objetivos de la Enfermería en Salud Ocupacional, donde el trabajador es su interés principal.

Del planteamiento antes señalado, se reconoce la necesidad de contribuir en la investigación de enfermería en el área de la salud laboral, al ser una fuerte oportunidad de 1) cuidar de la salud del trabajador, 2) establecer información actual de las condiciones con las que los trabajadores llegan o inician sus actividades laborales y cuáles desarrollan como consecuencia de la actividad laboral y 3) se empodera al personal de enfermería al visualizar la amplia responsabilidad que posee en ambientes como el de las distintas industrias.

Así, con base en lo anteriormente planteado es que se llevó a cabo la presente investigación con la finalidad de describir las características de salud y seguridad presentes en los trabajadores de una empresa automotriz de la ciudad de Querétaro, a través de un mapeo de riesgos utilizando el método FINE, con la finalidad de que se pueda contar con un referente actual del fenómeno que permita implementar estrategias de acción.

\section{Metodología}

Se trató de un estudio cuantitativo, de tipo descriptivo, transversal (Hernández Sampieri, Fernández Collado, \& Baptista Lucio, 2014), que se realizó en una empresa del giro automotriz, la cual se encuentra ubicada en la Ciudad de Querétaro, cuya población es de 76 trabajadores (de acuerdo a su registro de empleados con fecha de actualización al 02 de octubre de 2018), mismos que se dividen en dos áreas laborales, teniendo en primer instancia el área de venta automotriz, seguida de taller de mantenimiento y reparación, siendo esta última la zona evaluada en la que se cuenta con un total de 42 trabajadores.

El estudio se aprobó ante un Sub-Comité de Investigación y un Comité de Bioética, mismos que evaluaron los términos de los principios éticos, de 
acuerdo a los lineamientos de la Ley General de Salud en Materia de Investigación, así como del Código de Ética para Enfermería en México.

Los empleados fueron seleccionados por áreas debido a la vulnerabilidad de sufrir riesgos por el tipo de actividades que ahí realizan, aunado al cumplimiento de criterios de selección y que por supuesto, mediante el consentimiento informado aceptaran participar en el presente estudio.

En lo que respecta a los instrumentos, el mapeo de riesgos, resulta indispensable como herramienta de identificación de riesgos en los lugares de trabajo, puesto que mediante este se realiza un análisis de las condiciones laborales y del mismo modo se pueden crear proyectos con estrategias de mejora encaminadas a la prevención y progreso en las condiciones seguras del ambiente laboral. De lo anterior, se puede resumir que el SafeWork implementado por la OIT, es una metodología de la seguridad y salud en el trabajo para la evaluación y gestión de riesgos, la prevención de accidentes en el trabajo y enfermedades profesionales que contribuye a la promoción de una cultura de trabajo seguro (Falcón \& Rojas, 2017).

Los pasos para la metodología del mapeo se describen a continuación (SIMAPRO, 2019):

PASO N¹: Descripción del proceso de trabajo. Realizar la descripción detallada del proceso de trabajo, labores y operaciones. Cada etapa debe incluir el detalle de las labores que la constituyen e incluir al menos los elementos que integran el proceso de trabajo en el ingenio y la forma en que se realiza.

PASO N²: Identificación de los factores de riesgo. Recopilar por etapa y/o labor la siguiente información.

- $\quad$ Número de expuestos (\#Exp): Cantidad de trabajadores expuestos a los factores de riesgo en la labor.

- Tiempo de exposición (T-Exp): Cuantificar el tiempo real o promedio al que se exponen los trabajadores al factor de riesgo en la labor.

- Factor de riesgo: Identificar los tipos de riesgo a los que se exponen los trabajadores.

- Fuente generadora: Identificar de acuerdo a cada indicador, las fuentes que generan el riesgo a los trabajadores.

- Control: Identificar y describir las medidas de prevención y protección utilizadas para controlar los riesgos en la fuente, el medio y el trabajador.

- Posibles efectos: Identificar y describir los posibles efectos que pueden causar a la salud de los trabajadores los factores de riesgo y sus indicadores. 
PASO N³: Determinar el Grado de Riesgo (G.R.). Es la valoración cuantitativa de cada factor de riesgo identificado y se realiza aplicando la fórmula siguiente: G.R. $=\mathrm{C} \times \mathrm{E} \times \mathrm{P}$

- En donde: $\mathrm{C}=$ Consecuencia $\mathrm{E}=$ Exposición $\mathrm{P}=$ Probabilidad

PASO N4: Priorización de los factores de riesgo: Una vez obtenida la valoración de cada factor de riesgo, se procede a priorizarlos mediante los rangos indicados en la Figura $\mathrm{N}^{\circ} 1$.

PASO N5: Elaborar el mapa de factores de riesgo. Realizada la identificación de los riesgos, la valoración y la priorización de los factores de riego, se procede a elaborar el mapa de riesgos.

- El mapa debe abarcar todo el proceso y estar compuesto por símbolos y números indicando cuáles son los riesgos que más afectan a los trabajadores.

Figura 1. Representación de la priorización de los factores de riesgo

\begin{tabular}{|l|l|c|}
\hline \multicolumn{1}{|c|}{ RANGO } & \multicolumn{1}{|c|}{ TIPO DE RIESGO } & CLASIFICACION \\
\hline Mayor de 450 & Insoportable (6) & ROJO \\
\hline Mayor a 300 menor o igual a 450 & Extremo & ROJO \\
\hline Mayor a 200 menor o igual a 300 & Grave & ROJO \\
\hline Mayor a 100 menor o igual a 200 & Moderados (4) & AMARILLO \\
\hline Mayor a 30 menor o igual a 100 & Bajo & VERDE \\
\hline Menor o igual a 30 & Soportables (2) & VERDE \\
\hline
\end{tabular}

Fuente: SIMAPRO, 2019

PASO $\mathrm{N}^{\circ}$ 6: Plan de intervención. Basado en el mapa de factores de riesgo, se procede a definir e implementar las medidas de prevención y protección que garanticen a los trabajadores adecuadas condiciones de seguridad y salud.

\section{Resultados:}

La zona evaluada consta de un total de 42 individuos, siendo en su mayoría hombres, con un $71 \%$ (30) y el $29 \%$ (12) restantes son mujeres, los cuales se encuentran en un rango de 18 a 59 años de edad, teniendo una media de 36 años. La antigüedad laboral va desde los 6 meses hasta los 18 años. Cabe señalar que las actividades son desempeñadas en 7 áreas diferentes, teniendo un mayor porcentaje de trabajadores en el área de taller con un $48 \%$.

Respecto a los riesgos físicos, se incluyen diferentes características, donde $48 \%$ fueron de ruido, seguidas de las condiciones de las instalaciones con un 43\%, dirigidas estas últimas a la infraestructura de la empresa.

Para las características identificadas dentro del riesgo químico, se encontró que la exposición a combustibles representa un riesgo para el 57\% 
(24) de los trabajadores, 19\% (8) del personal indicó que se encuentra comprometido por otros tipos de químicos.

El riesgo mecánico fue referido por el 40\% (17) del personal operativo. Cabe destacar que el 36\% (15) de los mismos, reflejó encontrarse libre de sufrir daños a su salud y seguridad, no siendo así para los restantes 17\% (7) y $7 \%$ (3) de los empleados, puesto que las herramientas y equipos, respectivamente, son las fuentes principales de uso.

La estática postural despunta con un 71\% (30) de los trabajadores de la empresa automotriz. El personal, también dentro de sus actividades laborales, realiza cargas físicas y dinámicas, siendo estas últimas ejecutadas en un 24\% (10) y 5\% (2) respectivamente, por los empleados.

El 74\% (31) de los trabajadores del sector automotriz reflejan posible daño a la salud, secundado por el 21\% (9) de los mismos que presentan afección de tipo psicosocial. No menos importante, el 5\% (2) de los empleados, refiere una deficiente comunicación entre diferentes rangos jerárquicos.

\section{Clasificación por Método FINE}

De acuerdo a la valoración con respecto a los tres factores (Consecuencia - Probabilidad - Exposición) con los que el método FINE analiza la peligrosidad de los diferentes tipos de riesgos, se desglosan a continuación las gráficas que demuestran un puntaje relevante respecto de la probabilidad de presentar un daño a la salud y seguridad del personal.

Para los trabajadores del área de taller de mantenimiento y reparación, el método FINE arroja un puntaje grave (puntuación 4) con relación al riesgo físico (Figura 2), en donde se aprecia el uso de diferentes herramientas (pistola neumática, compresor, rampa de vehículos) y aspectos de la infraestructura de la empresa (pisos resbaladizos, áreas sin delimitar pasos de peatón).

Figura 2. Representación del riesgo físico por áreas

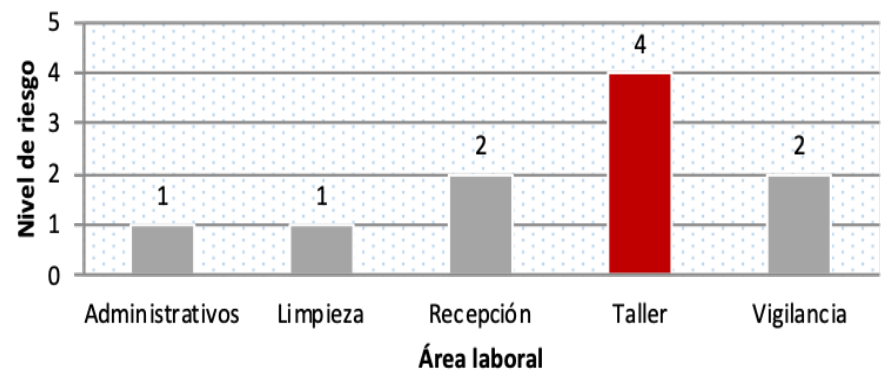

Fuente: recolección de datos, 2019.

Por otra parte, se pudo observar que las áreas de asesoría, refacciones y taller de mantenimiento-reparación se encuentran expuestas de manera grave (puntuación 4) a los riesgos del tipo químico (Figura 3); en el caso de los 
trabajadores de taller, se refirió exposición a dichos riesgos y otros más por las diferentes pruebas que realizan a los vehículos, ya que estos emanan gases como el monóxido de carbono.

Figura 3. Representación del riesgo químico por áreas

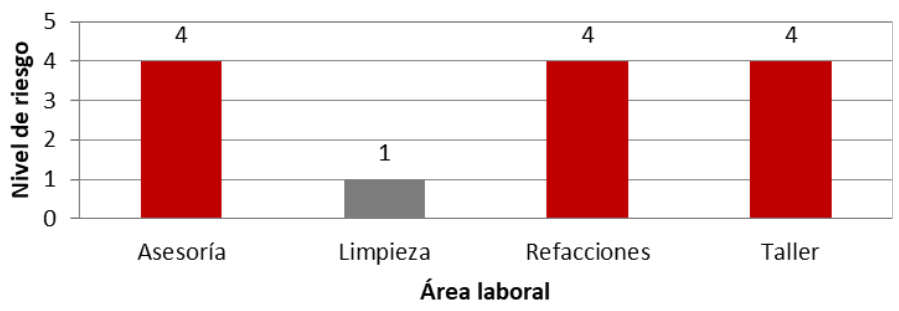

Fuente: recolección de datos, 2019

En lo que respecta al riesgo mecánico, se encontró una representación de un nivel moderado (puntuación 3) para el área de asesoría; los trabajadores de refacciones y propiamente de taller se encuentran expuestos a un bajo riesgo (puntuación 2).

El riesgo ergonómico se encuentra presente el todas las áreas (Figura 4), desde niveles soportables (puntuación 1) o bajos (puntuación 2), con excepción del sector de taller de mantenimiento, ya que de acuerdo al método FINE, se evaluó un nivel de riesgo grave (puntuación 4), en donde se observó la utilización de posturas y cargas diversas para la reparación de los vehículos. Al respecto, el personal manifestó dolores lumbares en primera instancia, seguidos de lesiones en miembros superiores.

Figura 4. Representación del riesgo ergonómico por áreas

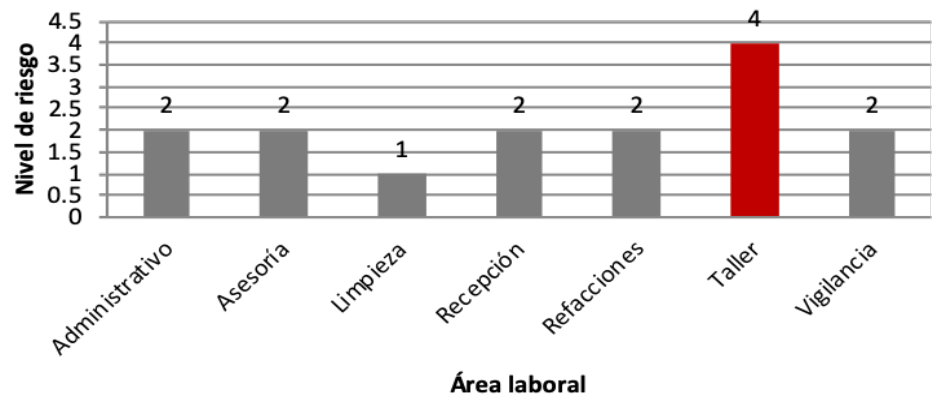

Fuente: recolección de datos, 2019.

De acuerdo a la clasificación FINE, el riesgo del tipo psicosocial predomina en el área de taller, valorándolo en riesgo grave debido a que se obtuvo una puntuación de 4, con características principales derivadas del ritmo de trabajo (Figura 5). 
Figura 5. Representación del riesgo psicosocial

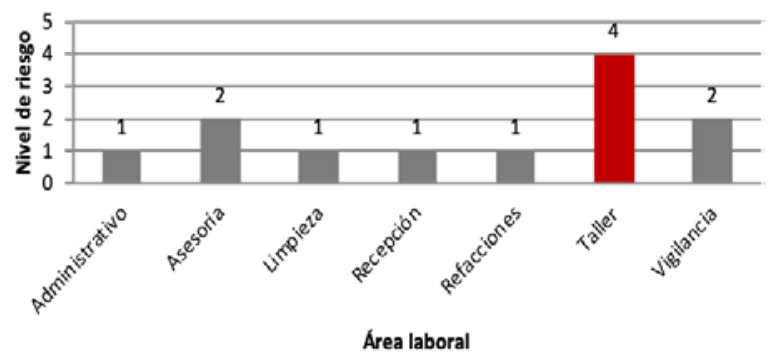

Fuente: recolección de datos, 2019.

\section{Conclusión y discusión:}

La aplicación de metodologías como el SafeWork, permite el fortalecimiento de programas enfocados a la seguridad de los trabajadores debido a las condiciones en las que realizan sus actividades, esta aplicación resulta sencilla y no requiere de largo tiempo para llevar a cabo los análisis necesarios para identificar los agentes causales de posibles daños a la salud. Asimismo, el apego a las normativas vigentes, coadyuva a una generación mayormente efectiva de programas preventivos con la finalidad de establecer las condiciones idóneas para desempeñar sus tareas laborales, sin exposición continua a riesgos.

Para la creación de medidas de prevención, enfocadas al cuidado de la integridad del trabajador, se hace necesaria la planeación y ejecución de acciones educativas, como capacitación para el trabajo, prácticas seguras laborales, entre otras, ya que la ausencia de estas, genera en gran medida, lo descrito anteriormente en los resultados de esta investigación.

En este sentido, cabe señalar que la evaluación de las características y riesgos laborales se han evidenciado de manera escasa en otras investigaciones, por lo que generar un contraste amplio con las mismas se torna complicado. No obstante, Lamberto expone que al realizar un análisis de siniestralidad, no sugiere a la problemática como una consecuencia de hechos aislados, sino que es el resultado del cómo se realizan los procesos y la estructura organizacional de la empresa para realizar el trabajo, por lo que describe también que, "la organización debe desarrollar formas eficaces de control, estableciendo sistemas que promuevan la confiabilidad de los componentes del proceso, en especial la confiabilidad humana. Es por ello que es altamente recomendable que la empresa trabaje en la implementación de sistemas de gestión de seguridad y salud ocupacional” (Lamberto, 2013).

En lo que respecta a México, la STPS señala que en la patología musculo-esquelética es de las primeras causas de morbilidad, tal como establece la evidencia de acuerdo a las estadísticas del IMSS del año 2012, en donde se reporta que el número de accidentes de trabajo de tipo músculo- 
esquelético fue de 240,674 casos (STPS, 2010). Seguido de esto, López, hace referencia a que estas patologías tienen un enorme y creciente impacto a nivel mundial desde la perspectiva de productividad y economía en el ámbito industrial, por lo que son la principal causa de dolor y discapacidad debido a su alta prevalencia y asociación con otras morbilidades, ocasionando un importante impacto socioeconómico (López Torres, González Muñoz, Colunga Rodríguez, \& Oliva López, 2014).

Por otra parte, en el estudio realizado en la Universidad de Loja (Obaco Pardo, 2015), se hace referencia a que una gestión adecuada de los riesgos existentes implica una toma de decisiones administrativas sumamente importantes; no obstante, las acciones que se lleven a cabo fortalecerán el control de dichos peligros, mismas que van a reducir el impacto que estos puedan tener en los trabajadores, ya sea físicos o biológicos. Posteriormente, en su apartado de señalización de seguridad, menciona que el propósito de establecer la señalética se hará con el principal objetivo de indicar fácilmente a los trabajadores la existencia de una situación que pueda poner en riesgo la salud del operador.

Del mismo modo, otro autor (Arana, 2015), evaluó cada uno de los riesgos existentes en el área automotriz, como ejemplo, menciona la valoración ergonómica para las mono-tareas más usadas en la labor mecánica mediante un método propuesto, que evalúa movimientos o esfuerzos repetidos de las manos que puedan afectar mano, muñeca y/o antebrazo, comparándolo con valores para los que se cree que la mayoría de los trabajadores pueden estar expuestos repetidamente sin sufrir efectos adversos para la salud.

Por otra parte, el estudio publicado por la OIT en 2016, "Prácticas de Trabajo Seguro en el Ingenio Pánuco", concuerda con la presente investigación al apuntar que la aplicación de la metodología de mapeo de riesgos, contribuye a la cultura de gestión de seguridad y salud ocupacional, donde destaca la reducción de los accidentes de trabajo y la gravedad de los mismos, la prevención de las enfermedades profesionales, y por consiguiente una disminución de costos indirectos y directos, pero sobre todo a una mejor calidad de la vida laboral de los trabajadores protegiendo su salud y sus vidas (Falcón, 2016).

Seguido de lo anterior, el estudio de Gestión de perfiles, mapas de riesgo y planes de acción para un Trabajo Seguro en la Industria Azucarera, publicado por la OIT en 2017 refiere que la transferencia de la metodología SafeWork en México a la agroindustria de la caña de azúcar y posteriormente a otros sectores como el turismo y PYME, en los últimos 3 años, demuestra la adaptabilidad, flexibilidad y eficiencia del método para la identificación de los factores de riesgo y su gestión preventiva en cualquier tipo de organización, coincidiendo con la investigación realizada, al señalar que la metodología de mapeo de riesgos, es de fácil aplicación y lenguaje sencillo y permite el trabajo 
en RED de intercambio de experiencias y gestión de conocimientos (Falcón \& Rojas, 2017).

Asimismo, es importante mencionar que resulta relevante abordar a los trabajadores desde un aspecto personal y familiar, puesto que estos factores resultan de importancia debido a que pueden potencializar la fuente generadora de un daño a la salud por causa de los diversos riesgos existentes en los lugares de trabajo.

Para finalizar, cabe mencionar que la enfermería ocupacional poco a poco va generando su lugar dentro de este campo laboral, debido a que actualmente en México, aún se encuentra poco reconocida, por lo que resulta importante seguir motivando al personal con investigaciones respecto al área, dando a conocer las posibilidades de implementar un modelo de cuidado con instrumentos propios y/o realizados por las diferentes organizaciones que se ocupan de los asuntos relativos al trabajo, que son fáciles de implementar para el personal capacitado.

Así mismo, cobra relevancia que el personal de enfermería se involucre con las diversas maneras, estrategias y herramientas, para valorar continuamente la salud del trabajador, no sólo en el aspecto biológico (lo involucrado con agentes patógenos), sino en aquello vinculado a las funciones que realizan (postura, ruido, u otro tipo de estresantes).

\section{References:}

1. Álvarez Fernández, S. (2015). Industria Automotriz, entre las más peligrosas para trabajar: IMSS. El Mejor Periodismo Diario, p. 1. Retrieved from https://pagina24.com.mx/local/2015/02/08/industriaautomotriz-entre-las-mas-peligrosas-para-trabajar-imss/

2. Arana, A. G. (2015). Análisis de seguridad e higiene en taller de reparación y mantenimiento Pire Tayen Automotores S.A. Universidad de la Fraternidad de Agrupaciones Santo Tomás de Aquino. http://redi.ufasta.edu.ar:8080/xmlui/bitstream/handle/123456789/142 6/2016_SH_043.pdf?sequence $=1$

3. Enciso Saldarriaga, A., López Sierra, M., \& Domínguez Ramírez, M. (2015). Cronología de la normatividad de los factores de riesgo psicosocial laboral en Colombia. Universidad CES.

4. Falcón, M. (2016). Prácticas de Trabajo Seguro en el Ingenio Pánuco. México. Retrieved from http:/www.coparmexchiapas.mx/herramientasoit/admin/57bb4b485f 52628aab93cbde76d322d0/df8c1e6ee4abbf31ffa6bef9bbc9efd5/c81e 728d9d4c2f636f067f89cc14862c_143.pdf

5. Falcón, M., \& Rojas, D. (2017). Gestión de perfiles , mapas de riesgo y planes de acción para un Trabajo Seguro en la Industria Azucarera. 
México.

Retrieved

from

https://www.gob.mx/cms/uploads/attachment/file/337922/Gesti_n_de _perfiles_mapas_de_riesgo_y_planes_de_acci_n_para_un_trabajo_s eguro_en_la_industria_azucarera.pdf

6. Federación, D. O. de la. (2018). Norma Oficial Mexicana NOM-035STPS-2018, Factores de Riesgo Psicosocial en el TrabajoIdentificación, Análisis y Prevención. Retrieved from https://dof.gob.mx/nota_detalle.php?codigo=5541828\&fecha=23/10/ 2018

7. Ferreras Remesal, A., López Ureña, A., Piedrabuena Cuesta, A., Oltra Pastor, A., \& Ruiz Folgado, R. (2011). Ergonomía y PYMES Especial referencia al sector de talleres de reparación de automóviles. Retrieved from

http://portal.ugt.org/saludlaboral/publicaciones/manual_estudio/MCA ERGONOMIA Y PYMES.pdf

8. González Sánchez, J. (2011). Aplicación del proceso de atención de enfermería a la salud laboral. Medicina y Seguridad Del Trabajo, 57(222), 15-22. Retrieved from http://scielo.isciii.es/pdf/mesetra/v57n222/especial2.pdf

9. Hernández Sampieri, R., Fernández Collado, C., \& Baptista Lucio, P. (2014). Metodología de la Investigación. (M. H. Interamericana, Ed.) (6ta ed.). México.

10. INSHT, I. N. de S. e H. en el T. (2015). Estrategia española de seguridad y salud en el trabajo. 2015-2020. Instituto nacional de seguridad e higiene en el trabajo. Madrid. Retrieved from http://www.insht.es/InshtWeb/Contenidos/Documentacion/ESTRAT EGIA SST 15_20.pdf

11. Lamberto, A. (2013). Análisis de la Siniestralidad en Empresa Automotriz. Universidad de Córdoba. Retrieved from https://rdu.unc.edu.ar/bitstream/handle/11086/826/P.I. FINAL Anabel Lamberto 2013.pdf?sequence=1\&isAllowed $=y$

12. López Torres, B. P., González Muñoz, E. L., Colunga Rodríguez, C., \& Oliva López, E. (2014). Evaluación de Sobrecarga Postural en Trabajadores: Revisión de la Literatura. Ciencia \& Trabajo, 16(50), 111-115. https://doi.org/10.4067/s0718-24492014000200009

13. Obaco Pardo, Á. E. (2015). Propuesta de reglamento, y un manual de seguridad industrial y salud ocupacional para la "Mecánica Industrial Aguirre” de la ciudad de Yanzatza. Universidad Nacional de Loja. Retrieved from https://dspace.unl.edu.ec/jspui/bitstream/123456789/8867/1/Ángel Eduardo Obaco Pardo.pdf

14. OIT. (2015). Seguridad y salud en el trabajo. Retrieved from 
http://ilo.org/global/topics/safety-and-health-at-work/lang-es/index.htm OMS. (2017). Protección de la salud de los trabajadores.

15. SafeWork. (2016). Gestión de perfiles y mapas de riesgo en seguridad y salud en el trabajo en la organización Presentación del SAFEWORK. Retrieved from http://www.herramientasoit.org/_lib/file/doc/Presentacion de la herramienta SAFEWORK.pdf

16. SIMAPRO-OIT. (2017). Promoviendo el Trabajo Seguro en la organización: perfiles, panoramas de riesgo y planes de acción (SafeWork). Retrieved

from https://www.oitsimapro.org/safework1.html

17. SIMAPRO. (2019). Metodologia de perfiles, panorama y mapa de riesgos. México. Retrieved from http://www.oitsimapro.org/uploads/3/1/9/0/31906627/metodologia_s afework.pdf

18. STPS. (2010). Información sobre Accidentes y Enfermedades de Trabajo Nacional. STPS. México. Retrieved from http://www.stps.gob.mx/bp/secciones/dgsst/estadisticas/Nacional 2001-2010.pdf

19. Zepeda García, E., \& García García, C. (2018). Universidad y Ciudad. Querétaro, ¿Ciudad Universitaria?Contraste Regional, 6(11),123-142. Retrievedfromhttp://revistacontrasteregional.ciisder.mx/index.php/rcr /article/view/61 